\title{
Changes in the incidence, prevalence and mortality of bronchiectasis in the UK from 2004 to 2013: a population-based cohort study
}

\author{
Jennifer K. Quint ${ }^{1,2}$, Elizabeth R.C. Millett ${ }^{2}$, Miland Joshi ${ }^{1}$, Vidya Navaratnam ${ }^{3}$, \\ Sara L. Thomas ${ }^{2}$, John R. Hurst ${ }^{4}$, Liam Smeeth ${ }^{2}$ and Jeremy S. Brown ${ }^{4}$
}

\begin{abstract}
Affiliations: ${ }^{1}$ Dept of Respiratory Epidemiology, Occupational Medicine and Public Health, National Heart and Lung Institute, Imperial College London, London, UK. ${ }^{2}$ Faculty of Epidemiology and Population Health, London School of Hygiene and Tropical Medicine, London, UK. ${ }^{3}$ Division of Epidemiology and Public Health, University of Nottingham, Nottingham, UK. ${ }^{4}$ Centre for Inflammation and Tissue Repair, UCL Respiratory, University College London, London, UK.
\end{abstract}

Correspondence: Jennifer K. Quint, Respiratory Epidemiology, Occupational Medicine and Public Health, G48, Emmanuel Kaye Building, Manresa Road, National Heart and Lung Institute, Imperial College London, London, SW3 6LR, UK. E-mail: j.quint@imperial.ac.uk

ABSTRACT There is a paucity of data on incidence, prevalence and mortality associated with non-cystic fibrosis bronchiectasis.

Using the Clinical Practice Research Datalink for participants registered between January 1, 2004 and December 31, 2013, we determined incidence, prevalence and mortality associated with bronchiectasis in the UK and investigated changes over time.

The incidence and point prevalence of bronchiectasis increased yearly during the study period. Across all age groups, the incidence in women increased from 21.2 per 100000 person-years in 2004 to 35.2 per 100000 person-years in 2013 and in men from 18.2 per 100000 person-years in 2004 to 26.9 per 100000 person-years in 2013. The point prevalence in women increased from 350.5 per 100000 in 2004 to 566.1 per 100000 in 2013 and in men from 301.2 per 100000 in 2004 to 485.5 per 100000 in 2013. Comparing morality rates in women and men with bronchiectasis in England and Wales ( $n=11862)$ with mortality rates in the general population from Office of National Statistics data showed that in women the ageadjusted mortality rate for the bronchiectasis population was 1437.7 per 100000 and for the general population 635.9 per 100000 (comparative mortality figure of 2.26). In men, the age-adjusted mortality rate for the bronchiectasis population was 1914.6 per 100000 and for the general population 895.2 per 100000 (comparative mortality figure of 2.14).

Bronchiectasis is surprisingly common and is increasing in incidence and prevalence in the UK, particularly in older age groups. Bronchiectasis is associated with a markedly increased mortality.

@ERSpublications

Bronchiectasis is increasing in incidence and prevalence in the UK, and is associated with an increased mortality http://ow.ly/Sh3Y9

For editorial comments see Eur Respir J 2016; 47: 10-13 [DOI: 10.1183/13993003.01567-2015].

This article has supplementary material available from erj.ersjournals.com

Received: June 302015 | Accepted after revision: Aug 202015 | First published online: Nov 052015

Support statement: J.K. Quint was funded on a Medical Research Council Population Health Scientist Fellowship (G0902135) and S.L. Thomas was funded by a National Institute for Health Research (NIHR) Career Development Fellowship (CDF 2010-03-32). University College London Hospitals/University College London received a proportion of funding from the Department of Health's NIHR Biomedical Research Centre's funding scheme. V. Navaratnam is funded by a NIHR Academic Clinical Fellowship. Funding information for this article has been deposited with FundRef.

Conflict of interest: Disclosures can be found alongside the online version of this article at erj.ersjournals.com

Copyright OERS 2016 


\section{Introduction}

Bronchiectasis is a long-term respiratory condition characterised by persistent airway infection and recurrent exacerbations in the presence of structurally abnormal bronchi. Although worldwide the commonest cause of bronchiectasis is previous acute lung infection, there is a large range of other causes and bronchiectasis could remain a relatively common condition even in high-income countries. However, there are few recent, comprehensive data on the epidemiology and associated mortality of bronchiectasis in a high-income country. No large study has been performed in the UK since the 1950s and there are only limited relevant data from non-UK sources [1-5]. Bronchiectasis can coexist and/or be associated with other long-term respiratory conditions, such as chronic obstructive pulmonary disease (COPD) and asthma, as well as many nonrespiratory diseases, including rheumatoid arthritis and HIV infection. Data on the coexistence of bronchiectasis with these diseases is lacking but is necessary to help inform management.

Using primary care data (Clinical Practice Research Datalink (CPRD)), we aimed to establish the incidence and prevalence of non-cystic fibrosis bronchiectasis in UK adults from 2004 to 2013. Additionally, we investigated the healthcare burden of bronchiectasis through mortality.

\section{Methods}

Data sources

CPRD is the world's largest validated computerised database of anonymised longitudinal medical records for primary care $[6,7]$. Data comprise $\sim 14$ million patients with around 5.4 million of these being currently alive and registered from 660 primary care practices spread throughout the UK. Records are derived from a widely used general practice software system and contain complete prescribing and coded diagnostic and clinical information as well as information on tests requested, laboratory results and referrals made at or following on from each consultation [8]. The population of patients within CPRD have been shown to be representative of the UK population with respect to age, sex and geographical distribution [9].

\section{Study population to determine incidence and prevalence of bronchiectasis}

Incidence was determined within financial years, with individuals aged $\geqslant 18$ years between April 1, 2004 and March 31, 2013 eligible for inclusion. To determine prevalence, the study population was the whole CPRD-Hospital Episode Statistics (HES) linked population over the age of 18 years enrolled in a CPRD practice on a specific date in each year between 2004 and 2013, stratified by age and sex.

\section{Bronchiectasis cohort study population and follow-up time}

A dynamic cohort of individuals who were alive and contributing to linked CPRD at any time between January 1, 2004 and December 31, 2013 and who had a diagnosis of bronchiectasis were identified using pre-specified Read codes (online supplementary material). We excluded individuals with coexisting Read codes for cystic fibrosis, to reduce misclassification of cystic fibrosis as non-cystic fibrosis bronchiectasis, as well as anyone aged $<18$ years at time of diagnosis of bronchiectasis and people who only had a record of bronchiectasis posthumously. Follow-up began at the latest of the following dates: study start date, the patient's 18th birthday, the date CPRD deemed the submitting general practice was "up to standard" (i.e. the practice had passed a number of quality checks and was deemed suitable for research use), the date a patient joined the practice plus 365 days, or the date of bronchiectasis diagnosis (for patients who received their first bronchiectasis code after start of the practice being up to standard or after January 1, 2004). The additional 365 days was added to the date the patient joined the practice, in order to exclude retrospective recording of historical bronchiectasis diagnoses reported when visiting a new general practitioner [10]. Follow-up ended at the earliest of the study end date, death, transfer out of the practice or the practice's last data collection date. Patients who contributed $\geqslant 1$ day of follow-up were included in the study.

\section{Codes used to define variables of interest}

Information on demographic factors (age at study start, sex, ethnicity and individual-level socioeconomic status), smoking status and other diseases associated with bronchiectasis (asthma, COPD, HIV, rheumatoid arthritis, other connective tissue diseases, inflammatory bowel disease, bone marrow transplant, hypogammaglobulinaemia and allergic bronchopulmonary aspergillosis (ABPA)) were obtained from the CPRD data using Read codes. Bronchiectasis was deemed to be idiopathic or post-infectious if it was not associated with any of the comorbidities listed above, and it was not possible within this database to further separate those two groups.

\section{Primary outcomes}

The outcome was incident or prevalent bronchiectasis, stratified by age, sex and year. 


\section{Statistical analyses}

All statistical analyses were conducted using Stata version 13 (StataCorp LP, College Station, TX, USA).

\section{Incidence and point prevalence}

Incidence rates were calculated by year, sex and age by dividing the number of new cases of bronchiectasis by the person-time at risk. Point prevalence was calculated by dividing the number of currently registered patients active in the dataset who had a bronchiectasis diagnosis on or before a randomly selected date (February 16 of each year, 2004-2013) by the total adult study population on that date. Age was grouped as those $<30$ years and then in 10 -year age bands up to 80 years, then as $\geqslant 80$ years.

\section{Cohort study}

The cohort of patients with prevalent bronchiectasis in the study period was used to estimate the prevalence of comorbid illnesses potentially associated with bronchiectasis. The presence of these comorbidities was ascertained both before and after the first bronchiectasis record.

\section{Mortality rates}

We calculated crude all-cause mortality rates by sex and 10-year age bands in the bronchiectasis population in 2010 by calculating the number of deaths in people with bronchiectasis in the linked CPRD-HES dataset in 2010 and dividing this by the mid-point bronchiectasis population in 2010. We used CPRD-HES linked patients only in this analysis. We compared results with Office of National Statistics published data on all-cause mortality figures for the same sex and age bands in England and Wales in 2010. Additionally, using direct standardisation, we calculated comparative mortality figures separately for men and women $[11,12]$.

\section{Ethics}

Ethics approval was obtained from ISAC (the Independent Scientific Advisory Committee overseeing CPRD (protocol 12_016)) and the London School of Hygiene and Tropical Medicine Ethics Committee. Following granting of ethics approval by ISAC, the length of the study was extended for a further 2 years (from the end of 2011 to 2013). This was considered a minor amendment and did not require further ISAC approval.

\section{Results}

\section{Incidence of bronchiectasis}

Across all age groups, the incidence of bronchiectasis in women increased overall from 21.24 per 100000 person-years in 2004 to 35.17 per 100000 person-years in 2013 and in men from 18.19 per 100000 person-years in 2004 to 26.92 per 100000 person-years in 2013. The details of age-specific rates are given in table 1. The incidence of bronchiectasis in men and women in 2004 and in 2013 increased with increasing age, except in men in 2013 in the $\geqslant 80$-years age group and in women aged $\geqslant 80$ years in 2004 and 2013 in whom the incidence decreased compared with the 70-79-year-old age groups (table 1). The yearly incidence of bronchiectasis stratified by age and sex from 2004 to 2013 is shown in figure 1 .

\section{Prevalence of bronchiectasis}

Across all age groups, the point prevalence of bronchiectasis in women increased from 350.5 per 100000 in 2004 to 566.1 per 100000 in 2013 and in men from 301.2 per 100000 in 2004 to 485.5 per 100000 in 2013. The details of prevalence by age are given in table 2. The recorded prevalence of bronchiectasis

TABLE 1 Incidence of bronchiectasis in 2004 and 2013 stratified by sex and age group

\begin{tabular}{|c|c|c|c|c|c|c|}
\hline \multirow{2}{*}{$\begin{array}{l}\text { Age } \\
\text { years }\end{array}$} & \multicolumn{2}{|c|}{ Men } & \multicolumn{2}{|c|}{ Women } & \multicolumn{2}{|c|}{ Overall } \\
\hline & 2004 & 2013 & 2004 & 2013 & 2004 & 2013 \\
\hline $18-29$ & $2.44(0.696-8.56)$ & $1.48(0.296-7.41)$ & $1.54(0.317-7.47)$ & $1.55(0.321-7.482)$ & $2.01(0.51-8.01)$ & $1.52(0.31-7.45)$ \\
\hline $30-39$ & $3.08(1.01-9.41)$ & $4.14(1.58-10.85)$ & $3.18(1.06-9.55)$ & $5.36(2.30-12.50)$ & $3.13(1.03-9.47)$ & 4.75 (1.93-11.68) \\
\hline $40-49$ & $7.74(3.29-15.66)$ & $9.28(4.88-17.66)$ & $8.95(4.65-17.23)$ & 13.63 (8.02-23.18) & $8.34(4.23-16.44)$ & $11.44(6.41-20.42)$ \\
\hline $50-59$ & $19.91(12.83-30.89)$ & $26.36(18.00-38.61)$ & $29.51(20.57-42.33)$ & $35.96(25.94-49.86)$ & $24.66(16.62-36.59)$ & 31.13 (21.91-44.24) \\
\hline $60-69$ & $49.68(37.62-65.60)$ & $58.06(44.90-75.09)$ & 60.55 (47.07-77.89) & 86.07 (69.69-106.3) & $55.17(42.38-71.82)$ & 72.31 (57.43-91.05) \\
\hline $70-79$ & 73.82 (58.77-92.73) & $126.66(106.43-150.74)$ & $66.32(52.14-84.36)$ & $124.94(104.86-148.87)$ & $69.72(55.14-88.16)$ & $125.74(105.59-149.74)$ \\
\hline$\geqslant 80$ & 77.68 (62.20-97.02) & $117.47(98.05-140.74)$ & $41.57(30.68-56.34)$ & 97.85 (80.27-119.28) & $54.02(41.38-70.52)$ & 105.38 (87.08-127.54) \\
\hline
\end{tabular}

Data are presented as incidence rate (95\% CI), per 100000 person-years. 

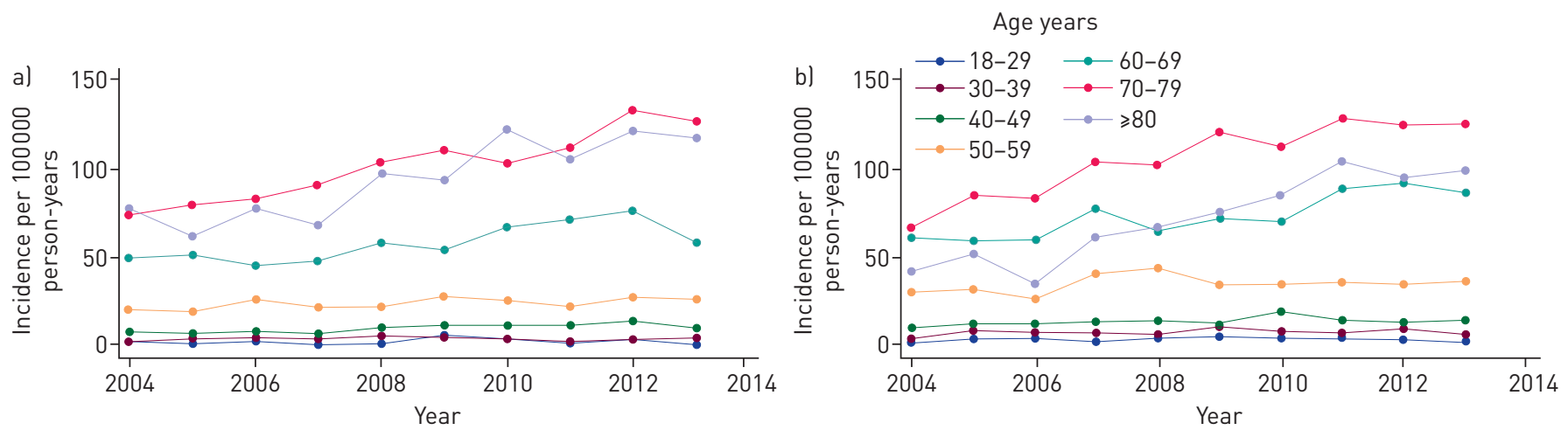

FIGURE 1 Incidence of bronchiectasis in the UK from 2004 to 2013 stratified by age in a) men and b) women.

increased over time within each age group in both men and women, remaining uncommon in those aged $<40$ years but reaching a very high prevalence in the elderly of around $1 \%$ by 2013 . The exception was the 50-59-year age group, in whom the prevalence of bronchiectasis slowly declined for both men and women over time (table 2 and figure 2). The recorded prevalence of bronchiectasis was higher in men in increasing age groups in the later years of the study and in women for all age groups except in those aged $\geqslant 80$ years (figure 2 ).

\section{Bronchiectasis cohort study population}

The bronchiectasis-diagnosed study population within CPRD consisted of 18793 individuals. Over half the individuals (58.5\%) were women, and the median age at diagnosis of adult bronchiectasis was 61.8 years (interquartile range 47.5-72.2 years). Among patients for whom socioeconomic status information was available (64\%), as measured by the Index of Multiple Deprivation (IMD), more patients belonged to the higher IMD quintile and numbers decreased with decreasing IMD. Bronchiectasis was more common in patients with higher socioeconomic status as measured by IMD (table 3 ).

\section{Coexisting comorbidities}

11914 (63.4\%) people had at least one coexisting comorbid illness associated with bronchiectasis. Asthma was the most prevalent coexisting comorbid illness, followed by COPD, then HIV infection, rheumatoid arthritis and connective tissue disorders (table 4). ABPA was only present in $1.8 \%$ of patients. Compared with those with comorbid illnesses, patients with idiopathic or post-infectious bronchiectasis were slightly older at the time of diagnosis (62.7 versus 60.0 years; $\mathrm{p}<0.001)$.

\section{Mortality rates}

Comparing age-specific mortality rates in men and women with bronchiectasis with age-specific mortality rates calculated in the general population in England and Wales from Office of National Statistics data, we found that in all age bands mortality rates were substantially higher in people with a diagnosis of bronchiectasis compared with the general population. The crude mortality rates by age group in 2010 per 1000 population are given in table 5 . Using direct standardisation, in women the age-adjusted mortality rate

TABLE 2 Prevalence of bronchiectasis in 2004 and 2013 stratified by sex and age group

\begin{tabular}{|c|c|c|c|c|c|c|}
\hline \multirow{2}{*}{$\begin{array}{l}\text { Age } \\
\text { years }\end{array}$} & \multicolumn{2}{|c|}{ Men } & \multicolumn{2}{|c|}{ Women } & \multicolumn{2}{|c|}{ Overall } \\
\hline & 2004 & 2013 & 2004 & 2013 & 2004 & 2013 \\
\hline $30-39$ & $38.7(28.3-53.0)$ & $58.2(45.0-75.2)$ & $53.4(40.8-69.8)$ & 79.3 (63.7-98.8) & $46.0(34.5-61.4)$ & $68.8(54.3-87.0)$ \\
\hline $40-49$ & 85.2 (68.9-105.3) & 97.4 (79.9-118.7) & 110.7 (91.9-133.3) & $142.1(120.6-167.4)$ & $98.0(80.4-119.4)$ & $119.8(100.2-143.2)$ \\
\hline $50-59$ & $286.9(255.6-322.0)$ & 224.0 (196.5-255.3) & 391.1 (354.3-431.7) & $285.9(254.6-321.0)$ & $339.0(304.8-377.0)$ & 254.9 (225.5-288.2) \\
\hline$\geqslant 80$ & $583.0(537.7-632.1)$ & 1236.9 (1170.2-1307.3) & $532.3(489.1-579.3)$ & $1175.6(1110.6-1244.3)$ & 557.7 (513.4-605.8) & $1206.3(1140.5-1275.8)$ \\
\hline
\end{tabular}

Data are presented as prevalence $(95 \% \mathrm{CI})$, per 100000 . 

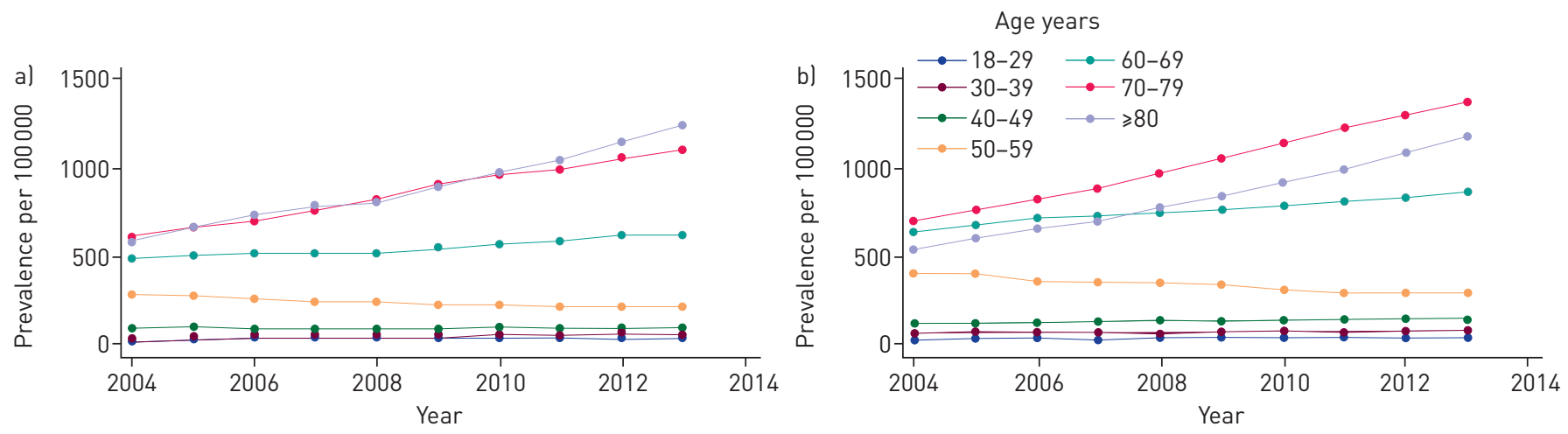

FIGURE 2 Prevalence of bronchiectasis in the UK from 2004 to 2013 stratified by age in a) men and b) women.

for the bronchiectasis population was 1437.7 per 100000 and for the general population 635.9 per 100000 (comparative mortality figure of 2.26). In men, the age-adjusted mortality rate for the bronchiectasis population was 1914.6 per 100000 and for the general population 895.2 per 100000 (comparative mortality figure of 2.14). This indicates that mortality for both men and women with bronchiectasis is more than twice the mortality in the general population, independent of age differences between the two populations.

\section{Discussion}

This study provides detailed estimates of the recorded incidence and prevalence of bronchiectasis in the UK over time, the prevalence of associated diseases, and an assessment of the burden on healthcare resources in terms of mortality. Data from the USA and Germany and previous data from the UK have all suggested that the clinical importance of bronchiectasis is increasing, with increases in prevalence [3, 13], hospital admissions [4] and mortality [5]. Our data provide additional insight beyond these studies, with the large number of patients included in the cohort and the long study period enabling us to give accurate estimates of bronchiectasis incidence and prevalence stratified by age groups and sex. Although general practices are self-selecting with regard to contributing to CPRD, they are broadly representative of the UK population [9], and previous studies have established the validity of medical diagnoses [14, 15] and prescribing records in CPRD [16]. Hence, the cohort of patients included in our study is likely to be representative of patients with a diagnosis of bronchiectasis in their medical records across the UK. Overall, this study provides important and previously unavailable information regarding bronchiectasis in the UK. The data are also likely to be representative for trends in other industrialised countries and are in keeping with data from a previous British Thoracic Society bronchiectasis audit [17].

TABLE 3 Baseline characteristics of the bronchiectasis study population

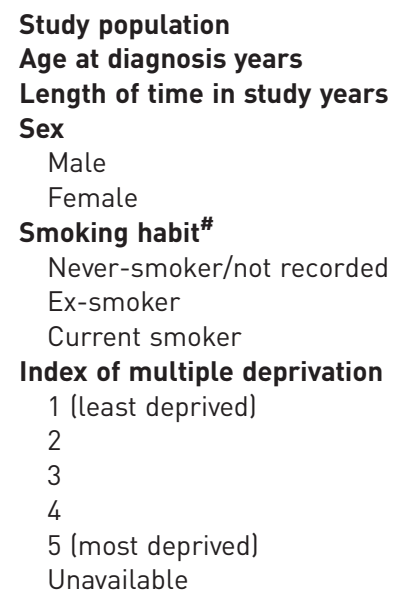

Data are presented as $\mathrm{n}$, median (interquartile range) or $\mathrm{n}(\%) .{ }^{*}$ : ever, closest to diagnosis date. 
TABLE 4 Coexisting diagnoses associated with bronchiectasis $\#$

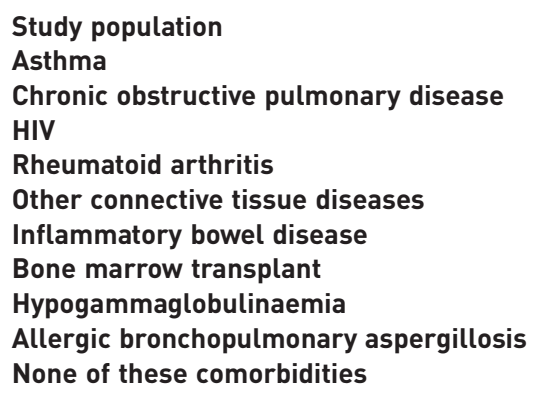

Data are presented as $\mathrm{n}$ or $\mathrm{n}(\%) .{ }^{\#}$ : some patients had multiple comorbidities.

The advent of antibiotics and improved standards of living in industrialised countries has led to large reductions in severe childhood respiratory tract infections, perhaps leading to an assumption that bronchiectasis was a disease of the past in these countries. However, we found that the recorded incidence and prevalence of bronchiectasis in the UK has increased year on year during the study period, with an increase in almost all age groups but with the most rapid increase seen in women aged $>70$ years. In the older population, bronchiectasis was a common disease affecting $>1 \%$ of both men and women aged $>70$ years. Bronchiectasis was also surprisingly common in younger adults as well, with a prevalence in 2013 of $>40$ per 100000 even in those aged $<30$ years. The exception for the increasing prevalence of bronchiectasis in the UK over time is the 50-59-year-old group, for which prevalence slowly declined between 2004 and 2013. The reasons for this discrepancy are not clear. One potential explanation is that a proportion of bronchiectasis in the 50-59-year-old age group is due to childhood disease that has become less common with time. In contrast, the increase in prevalence of bronchiectasis in older age groups is driven by adult-onset causes of bronchiectasis that are increasingly affecting older age groups.

The reasons for the overall increased incidence and prevalence of bronchiectasis are not clear. The increases may be due to changes in the prevalence of causes of bronchiectasis, but may also be due to improved diagnosis rates, with increased use of computed tomography (CT) scans to assess patients with lung disease. It is also possible that changes in diagnostic labelling with respect to other associated respiratory diseases have contributed to the increased incidence. Comparing these data with published data available on other respiratory diseases, the prevalence of COPD has increased by 50\% from 2000 to 2009 but incidence has remained static [18]. Overall, with bronchiectasis, the increased incidence and prevalence over time is likely to be at least partly driven by increased case ascertainment and increased investigation of the elderly population. Nonetheless, age-specific mortality rates were higher in people with bronchiectasis than the general population (including in younger patients), indicating that a diagnosis of bronchiectasis is associated with important health effects. Whether the increased mortality in the bronchiectasis cohort is due to complications from coexisting illnesses or directly attributable to bronchiectasis is not known. We were only able to investigate all-cause mortality. It is not clear from these data whether the increased mortality in people with bronchiectasis was due to nonrespiratory causes, or indeed whether mortality was higher in those with comorbidities. More studies are needed to characterise the health burden associated with bronchiectasis by investigating general practice visits for exacerbations and hospital admissions, and to investigate the mechanisms of the increased mortality of patients with bronchiectasis.

\section{TABLE 5 Crude mortality rates by age group in 2010}

\begin{tabular}{lccccc}
\multirow{2}{*}{ Age years } & \multicolumn{2}{c}{ Men } & & \multicolumn{2}{c}{ Women } \\
\cline { 2 - 3 } \cline { 5 - 6 } & General population & Bronchiectasis cohort & & General population & Bronchiectasis cohort \\
\hline $\mathbf{1 8 - 4 9}$ & $1.3(1.3-1.4)$ & $13.1(3.4-22.8)$ & & $0.8(0.7-0.8)$ & $6.4(0.8-12.0)$ \\
$\mathbf{5 0 - 5 9}$ & $5.1(5.0-5.2)$ & $10.0(2.6-17.3)$ & & $3.4(3.4-3.5)$ & $7.8(2.4-13.2)$ \\
$\mathbf{6 0 - 6 9}$ & $12.5(12.3-12.6)$ & $29.5(20.6-38.4)$ & & $7.9(7.8-8.0)$ & $16.0(10.6-21.5)$ \\
$\mathbf{7 0 - 7 9}$ & $33.77(33.4-33.9)$ & $58.6(46.4-70.7)$ & & $22.8(22.6-23.0)$ & $43.9(34.9-52.8)$ \\
$\geqslant \mathbf{8 0}$ & $111.8(111.1-112.5)$ & $144.6(115.4-173.9)$ & & $98.9(98.4-99.4)$ & $160.1(136.1-184.1)$ \\
\hline
\end{tabular}

Data are presented as mortality rate $(95 \% \mathrm{CI})$, per 1000 population. Rates have been calculated using a mid-year population estimate for 2010. 
Bronchiectasis has a significant sex bias, being more common in women than in men. Bronchiectasis has a wide range of causes and associated comorbidities. These include both asthma and irreversible airways obstruction, which, given the spirometric criteria used to diagnose COPD in the UK, could be misdiagnosed as COPD. Conversely, bronchiectasis is increasingly recognised as a complication of asthma and COPD [19-21]. In our cohort of patients with bronchiectasis, both asthma and COPD were very common, associated with $43 \%$ and $36 \%$ of subjects, respectively. It is unclear in what proportion of these patients the diagnosis of bronchiectasis was secondary to the airways disease rather than the primary disease. One interesting finding was the fact that asthma-associated bronchiectasis was more common than COPD-associated bronchiectasis. While some of this may be due to misclassification of COPD as asthma in the database, it warrants further investigation. The low prevalence of ABPA-associated bronchiectasis in this cohort is probably due to under-recording of ABPA in primary care. One new and unexpected finding was the relatively high proportion of patients with bronchiectasis who were HIV positive (6.9\%), had rheumatoid arthritis or another connective tissue disease (11.4\%), or inflammatory bowel disease (2.8\%). Although these conditions are known causes of bronchiectasis, they were not prominent contributors in previous case series of bronchiectasis associations from secondary or tertiary referral centres [22]. According to national figures, the estimated prevalence of HIV is $\sim 1.5$ per 1000 of the UK population [23]. The increase in the association of HIV infection, rheumatoid arthritis/connective tissue disease and inflammatory bowel disease with bronchiectasis in our cohort may reflect the strength of population studies for establishing more accurate information on disease associations. We believe that the CPRD population is representative and not richer in HIV than expected. It is likely that more CT scans are performed in the HIV population and so the rate of bronchiectasis is higher in this group. This may mean that the rate of bronchiectasis in this group is actually more accurate than in the non-HIV group.

\section{Limitations}

In order to improve sensitivity and specificity of the diagnosis, only a small number of specific Read codes were used to identify subjects with bronchiectasis, and individuals had to be aged $>18$ years when they were first diagnosed. As a consequence, some patients with bronchiectasis would have been excluded. Bronchiectasis was deemed to be idiopathic if it was not associated with any of the comorbidities previously listed. While some of the bronchiectasis codes could be post-infectious, we included individuals with such codes in the idiopathic bronchiectasis group as these codes were only used in $2 \%$ of our study population and over half of those patients also had a Read code for "Bronchiectasis" (see supplementary material). We are unable to confirm that the diagnosis of bronchiectasis in the general practice records is accurate. We did not have access to CT scan data and therefore cannot be sure that in each recorded case of bronchiectasis the diagnosis was attained according to current guidelines. However, as the diagnosis is usually made in a secondary care setting and requires a CT scan $[17,24]$, it is unlikely that a diagnosis of bronchiectasis will be recorded by the general practitioner without confirmation from secondary care.

\section{Conclusions and clinical implications}

There are major deficits in our understanding of the pathophysiology and management of bronchiectasis [25], yet our data show that bronchiectasis is increasingly common and is associated with a substantially raised mortality. Bronchiectasis remains an important cause of respiratory disease in the UK and this should be reflected in the provision of clinical care for these patients.

\section{References}

1 Seitz AE, Olivier KN, Steiner CA, et al. Trends and burden of bronchiectasis-associated hospitalizations in the United States, 1993-2006. Chest 2010; 138: 944-949.

2 Loebinger MR, Wells AU, Hansell DM, et al. Mortality in bronchiectasis: a long-term study assessing the factors influencing survival. Eur Respir J 2009; 34: 843-849.

3 Seitz AE, Olivier KN, Adjemian J, et al. Trends in bronchiectasis among Medicare beneficiaries in the United States, 2000 to 2007. Chest 2012; 142: 432-439.

4 Ringshausen FC, de Roux A, Pletz MW, et al. Bronchiectasis-associated hospitalizations in Germany, 2005-2011: a population-based study of disease burden and trends. PLoS One 2013; 8: e71109.

5 Roberts HJ, Hubbard R. Trends in bronchiectasis mortality in England and Wales. Respir Med 2010; 104: 981-985.

6 The Clinical Practice Research Datalink. www.cprd.com Date last accessed: February 28, 2015.

7 Williams T, van Staa T, Puri S, et al. Recent advances in the utility and use of the General Practice Research Database as an example of a UK Primary Care Data resource. Ther Adv Drug Saf 2012; 3: 88-99.

8 Tate AR, Beloff N, Al-Radwan B, et al. Exploiting the potential of large databases of electronic health records for research using rapid search algorithms and an intuitive query interface. J Am Med Inform Assoc 2014; 21: 292-298.

9 Herrett E, Gallagher AM, Bhaskaran K, et al. Data Resource Profile: Clinical Practice Research Datalink (CPRD). Int J Epidemiol 2015; 44: 827-836.

10 Lewis JD, Bilker WB, Weinstein RB, et al. The relationship between time since registration and measured incidence rates in the General Practice Research Database. Pharmacoepidemiol Drug Saf 2005; 14: 443-451. 
11 Office for National Statistics. Death Registrations Summary Tables, England and Wales, 2010. www.ons.gov.uk/ ons/publications/re-reference-tables.html?edition=tcm\%3A77-227638. Date last updated: July 13, 2011. Date last accessed: March, 2015.

12 Office for National Statistics. Population Estimates for UK, England and Wales, Scotland and Northern Ireland, Mid-2001 to Mid-2010 Revised. www.ons.gov.uk/ons/publications/re-reference-tables.html?edition=tcm\%3A77315018 Date last updated: December 17, 2013. Date last accessed: March, 2015.

13 Weycker D, Edelsberg J, Oster G, et al. Prevalence and economic burden of bronchiectasis. Clin Pulm Med 2005; 4: 205-209.

14 Hansell A, Hollowell J, Nichols T, et al. Use of the General Practice Research Database (GPRD) for respiratory epidemiology: a comparison with the 4th Morbidity Survey in General Practice (MSGP4). Thorax 1999; 54: 413-419.

15 Herrett E, Thomas SL, Schoonen WM, et al. Validation and validity of diagnoses in the General Practice Research Database: a systematic review. Br J Clin Pharmacol 2010; 69: 4-14.

16 Hollowell J. The General Practice Research Database: quality of morbidity data. Popul Trends 1997; 87: 36-40.

17 Pasteur MC, Bilton D, Hill AT. British Thoracic Society guideline for non-CF bronchiectasis. Thorax 2010; 65: Suppl. 1, i1-i58.

18 James G, Petersen I, Donaldson G, et al. Longitudinal changes in the rate and mean age of incidence and prevalence of COPD in the UK, 2000-2009. Thorax 2011; 66: Suppl. 4, A154.

19 Martínez-García MA, Soler-Cataluña JJ, Donat Sanz Y, et al. Factors associated with bronchiectasis in patients with COPD. Chest 2011; 140: 1130-1137.

20 Wedzicha JA, Hurst JR. Structural and functional co-conspirators in chronic obstructive pulmonary disease exacerbations. Proc Am Thorac Soc 2007; 4: 602-605.

21 Ip MS, So SY, Lam WK, et al. High prevalence of asthma in patients with bronchiectasis in Hong Kong. Eur Respir J 1992; 5: 418-423.

22 Pasteur MC, Helliwell SM, Houghton SJ, et al. An investigation into causative factors in patients with bronchiectasis. Am J Respir Crit Care Med 2000; 162: 1277-1284.

23 Public Health England. HIV in the United Kingdom: 2013 report. PHE publications gateway number: 2013256 London, Public Health England, 2013. Available from: www.gov.uk/government/collections/hiv-surveillance-dataand-management

24 Hill AT, Routh C, Welham S. National BTS bronchiectasis audit 2012: is the quality standard being adhered to in adult secondary care? Thorax 2014; 69: 292-294.

25 De Soyza A, Brown JS, Loebinger MR. Research priorities in bronchiectasis. Thorax 2013; 68: 695-696. 